\title{
Enkephalin Knockdown in the Basolateral Amygdala Reproduces Vulnerable Anxiety-Like Responses to Chronic Unpredictable Stress
}

\author{
Patrick Bérubé', Jean-François Poulin', Sylvie Laforest' and Guy Drolet*, \\ 'Centre de recherche du CHU de Québec, Axe Neurosciences, Université Laval, Quebec, QC, Canada
}

\begin{abstract}
The endogenous enkephalins (ENKs) are potential candidates participating in the naturally occurring variations in coping styles and determining the individual capacities for adaptation during chronic stress exposure. Here we demonstrate that there is a large variance in individual behavioral, as well as in physiological outcomes, in a population of Sprague-Dawley rats subjected to 3 weeks of chronic unpredictable stress (CUS). Separation of resilient and vulnerable subpopulations reveals specific long-term neuroadaptation in the ENKergic brain circuits. ENK mRNA expression was greatly reduced in the posterior basolateral nucleus of amygdala (BLAp) in vulnerable individuals. In contrast, ENK mRNA levels were similar in resilient and control (unstressed) individuals. Another group of rats were used for lentiviralmediated knockdown of ENK to assess whether a decrease of ENK expression in the BLAp reproduces the behavioral disturbances found in vulnerable individuals. ENK knockdown specifically located in the BLAp was sufficient to increase anxiety in the behavioral tests, such as social interaction and elevated plus maze when compared with control individuals. These results show that specific neuroadaptation mediated by the ENKergic neurotransmission in the BLAp is a key regulator of resilience, whereas a decrease of the ENK in the BLAp is a maladaptation mechanism, which mediates the behavioral dichotomy observed between vulnerable and resilient following 3 weeks of CUS. Neuropsychopharmacology (2014) 39, I 159-1 168; doi:I0.1038/npp.2013.316; published online II December 2013
\end{abstract}

Keywords: opioid; amygdala; shRNA; chronic stress; resilience

\section{INTRODUCTION}

Resilience to chronic stress exposure emerges through various forms of gene-environment interactions. Recent studies demonstrated some aspects of the neurobiological mechanisms associated with stress resistance and vulnerability (Krishnan et al, 2007; Bergstrom et al, 2008; Wood et al, 2010; Covington et al, 2010; Taliaz et al, 2011; Christiansen et al, 2012; Wang et al, 2012), and suggested that mechanisms underlying resilience is mediated by adaptive changes in several neural circuits involving numerous neurotransmitters and molecular pathways (Feder et al, 2009). Among the neuropeptide systems, the endogenous opioid enkephalins (ENKs) are very interesting candidates to participate in the naturally occurring variations in coping styles and to determine the individual capacity for adaptation during chronic stress exposure. The most consistent finding among studies using different species and stressors is that the ENKergic neurotransmission, through mu-opioid receptors ( $\mu$-ORs) and/or delta-OR

*Correspondence: Dr G Drolet, Centre de recherche du CHU de Québec, Axe Neurosciences, Université Laval, CHUL P-09800, 2705 Laurier, Québec, QC, Canada GIV 4G2.

Tel: + 4l8525 4444, ext. 47979, Fax: + 4186542753 ,

E-mail: guy.drolet.2@ulaval.ca

Received 19 July 20 I3; revised 16 October 20 I3; accepted I 8 October 2013; accepted article preview I| November 2013
( $\delta$-ORs), is critical in different aspects of affective states, basal hedonic state, and attenuation of stress-induced neuroendocrine, autonomic, and anxiety-like behavioral responses (König et al, 1996; Kang et al, 2000; Filliol et al, 2000; Ragnauth et al, 2001; Drolet et al, 2001; Hebb et al, 2004; Hebb et al, 2005a; Hebb et al, 2005b; Jutkiewicz, 2007; Noble et al, 2007). In the present study, we assessed whether changes in the expression of ENK in 23 nuclei of the basal forebrain are associated to resilience or vulnerability to chronic unpredictable stress (CUS). In the first experiment, physiological and behavioral alterations induced by the CUS model were assessed. The results emerging from this work demonstrate that there is significant physiological and behavioral variability among an otherwise homogenous population of chronically stressed rats. We found that resilience or vulnerability to CUS was associated with specific ENKergic long-term neuroadaptation in specific brain circuit. In the second experiment we tested whether the knockdown of ENK in the basolateral nucleus of amygdala (BLAp) induced anxiety-like behavioral responses as seen in vulnerable individuals.

\section{MATERIALS AND METHODS}

\section{Housing Condition and General Procedure}

Male Sprague-Dawley rats weighing 250-300 $\mathrm{g}$ (Harlan Laboratories, Indianapolis, IN) were housed individually 
in a standard cage 14 days before testing under a 12-h light/ dark cycle, the rats were provided ad libitum chow and water. Two experimental designs were used in this study: (1) the CUS model was used to evaluate whether the expression of ENK in the prefrontal cortex, dorsal striatum, nucleus accumbens (NAc), and amygdaloid complex are associated to the behavioral responses among resilient and vulnerable individuals; and (2) lentiviral-mediated knockdown of ENK was bilaterally induced in the BLAp to assess whether a decrease of ENK in this nucleus produced the anxiety-like responses found in vulnerable individuals. Research protocols and animal care conformed to the guiding principles for animal experimentation as enunciated by the Canadian Council on Animal Care and approved by the Ethical Committee of Université Laval for Animal Research. All efforts were made to minimize animal pain, discomfort, or suffering, and the number of rats used.

\section{Experiment 1}

The CUS stress model. The CUS regimen used was adapted from that previously described by Bondi et al (2007). The daily stressors were used in a semi-random sequence at varying times during the day, over the course of 21 days in the stress group $(n=48)$. The complete schedule detailing the order of the different stressors and behavioral tests is presented in Table 1. All animals (stressed and unstressed) were weighed daily and exposed to behavioral tests. In order to balance the groups, rats were assigned to either a control or an experimental group on the basis of their initial weight, sucrose intake and preference, and social interaction score. Unfortunately, we did not have the initial social interaction data for all the rats. Therefore, these rats were matched on the other parameters. The control rats $(n=25)$ were individually housed for the same period of time. All animals were euthanized 7 days after the last stressor.

Sucrose intake and preference. Animals were habituated to sucrose $(1 \%)$ or water bottles in the homecage for 14 days. The measures were taken by pre-weighing water and sucrose solution-filled bottles. Animals were given the choice between two bottles for $4 \mathrm{~h}$, from 1700 to 2100 hours, and the bottles were weighted post consumption. The position of bottles was switched daily to avoid a place preference. Sucrose measurements were taken before, during, and after the exposition to CUS (Table 1).

Social interaction and elevated plus maze. The social interaction and elevated plus maze (EPM) tests were performed as described in Poulin et al (2013). Animals were habituated twice (one time per day, 2 days before the day of testing) to behavioral room for $15 \mathrm{~min}$ and than to the social interaction arena for $5 \mathrm{~min}$. Social interaction was performed 4 days before and 3 days after the exposition of CUS. The EPM was only performed 4 days after the last exposition of CUS (Table 1). An acclimatization period to the behavioral room of $15 \mathrm{~min}$ was also provided on the day of each test. The social interaction evaluated the number and the time spent sniffing, chasing, or grooming by the experimental animals on a novel interaction partner with no more than $10 \%$ weight difference. The interactive rats were allowed to interact no more than three times, each time was separated by at least $1 \mathrm{~h}$. The EPM evaluates the open-arm exploration, independent of any potential changes in total exploration or locomotion, was the open/total ratio, defined as the time spent in the open arms as a proportion of time spent in all four arms. Videos were recorded and analyzed with the ANY-maze software by an investigator blind to the treatment group of the subjects.

Assay of plasma corticosterone. Two blood samples were collected through the lateral saphenous vein before and immediately following the 30 -min restraint sessions to measure plasma corticosterone levels. Unstressed animals also received venipunctures without restrain stress, thus each sample represents the basal condition. To minimize the stress induced by the sampling procedure, animals were handled 3 days before. During handling, animal legs were shaved and animals were habituated to a short restraint. For blood sampling, the saphenous vein was stabbed with a needle (22 gauge), and the blood was collected in a micro-hematocrit tube (CB300; Sarstedt, Montréal, QC, Canada). Corticosterone levels were determined by radioimmunoassay using the same condition as described in Dumont et al (2000).

Perfusion, tissue processing, and radioactive in situ hybridization. Perfusion and tissue processing were performed as described in previous investigations (Bérubé et al, 2013; Poulin et al, 2006, 2008, 2013). The cRNA probe directed against ENK (938 bp) was generated from a plasmid generously provided by $\mathrm{Dr}$ Sabol and target nucleotide -104 to 832 . Brain slides were revealed after $16 \mathrm{~h}$ on KODAK BioMax MR Film (Kodak, Rochester, NY, USA). Films were exposed with a Precision illuminator B95 (Imaging Research, St Catherines, ON, Canada) during images capture. Autoradiographic images were digitally acquired with a Retiga-2000R camera (QImaging, Surrey, BC, Canada) with QCapture software. Optical density was quantified with ImageJ software and normalized with $\mathrm{C}^{14}$ standard slides (American Radiolabeled Chemicals, St Louis, MO, USA). Structure used for in situ hybridization are listed in Supplementary Table S1. Representative autoradiographic images and schematic drawing of areas in which ENK was quantified are presented in Figure 1. An area of $0.5 \times 0.4 \mathrm{~mm}$ was used to quantify mRNA in striatum, NAc, and amygdaloid complex, and an area of $0.1 \mathrm{~mm} \times 0.5 \mathrm{~mm}$ was used in prefrontal cortex. Analysis was performed without knowledge of the rat's condition.

\section{Experiment 2}

Vectors construction, lentivirus production, and stereotaxic surgery. The shRNA expression vectors were designed to produce a shRNA targeting the ENK or a scrambled mRNA sequences, and the lentivirus production methods were produced as described in Poulin et al (2013). The titer of the viral preparation used in this study ranged from 1.0 to $8.0 \times 10^{8} \mathrm{TU} / \mathrm{ml}$. Twenty-three rats were deeply anesthetized under isoflurane (2\%) and placed in the stereotaxic apparatus. The viral preparations were injected into the BLAp using a Hamilton neuros syringe (7002 KH SYR $2 \mu \mathrm{l}$ ). The stereotaxic coordinates for the BLAp were 
Table I Chronic Unpredictable Stress Experimental Schedule

\begin{tabular}{|c|c|c|c|c|}
\hline Days & Treatment & AM/PM & $\begin{array}{l}\text { Sucrose } \\
\text { intake and } \\
\text { preference }\end{array}$ & $\begin{array}{c}\text { Blood } \\
\text { sampling }\end{array}$ \\
\hline-12 to -4 & Sucrose habituation & & & \\
\hline-4 & Social interaction & AM & & \\
\hline-3 to -1 & & & $x$ & \\
\hline । & 30 min restraint & AM & & $x$ \\
\hline 2 & 15 min footshock & PM & & \\
\hline 3 & $24 \mathrm{~h}$ wet bedding & AM & & \\
\hline 4 & $24 \mathrm{~h}$ density housing & AM & & \\
\hline 5 & 15 min warm water swim & PM & & \\
\hline 6 & 15 min footshock & AM & & \\
\hline 7 & $24 \mathrm{~h}$ density housing & AM & & \\
\hline 8 & 15 min warm water swim & PM & & \\
\hline 9 & I h shaking/crowding & AM & $x$ & \\
\hline 10 & 10 min cold water swim & PM & $x$ & \\
\hline ।1 & 30 min restraint & AM & & $x$ \\
\hline 12 & 15 min footshock & PM & & \\
\hline 13 & $24 \mathrm{~h}$ density housing & AM & & \\
\hline 14 & I h shaking/crowding & AM & & \\
\hline 15 & $24 \mathrm{~h}$ wet bedding & AM & & \\
\hline 16 & 15 min footshock & PM & & \\
\hline 17 & $24 \mathrm{~h}$ density housing & AM & & \\
\hline 18 & $24 \mathrm{~h}$ wet bedding & AM & & \\
\hline 19 & I h shaking/crowding & AM & $x$ & \\
\hline 20 & 10 min cold water swim & PM & $x$ & \\
\hline 21 & 30 min restraint & AM & & $x$ \\
\hline+3 & Social interaction & AM & & \\
\hline+4 & Elevated plus maze & AM & & \\
\hline+4 to +6 & & & $x$ & \\
\hline+7 & Euthanized & AM & & \\
\hline
\end{tabular}

The ' $x$ ' indicate when the sucrose intake and preference and blood sampling were done.

AP: -2.8 , ML: 4.8, and DV: -9.0 . After a waiting time of $5 \mathrm{~min}$, the virus was injected at a dosage of $0.1 \mu \mathrm{l} / \mathrm{min}$ and the injection was followed by another 5 min waiting period. Fifteen rats were injected bilaterally with $1 \mu \mathrm{l}$ of ENK shRNA and eight rats were injected with scrambled shRNA. Animals were single-housed and given at least 14 days (recovery period) to ensure sufficient time for the animal to recover after the surgery, as well as for optimal vector expression and reduction of ENK level. Each rat was weighed daily and exposed to behavioral tests. Social interaction was assessed 3 days before the surgery and 3 days following the recovery period. Sucrose intake was assessed during 3 days, starting 3 days before the beginning of the surgery and 4 days following the recovery period. EPM was performed $24 \mathrm{~h}$ after the final social interaction test.

Localization of injection sites by copepod green fluorescent protein immunohistochemistry and quantification of the knockdown. The shRNA expression vectors expressed the copepod green fluorescent protein (copGFP). Localization of the neurons transduced by the lentivirus was evaluated by a copGFP immunohistochemistry. Briefly, brain sections were washed in sterile PBS, 30 min blocking step $(0.3 \%$ Triton X-100 and $1 \%$ BSA in PBS, pH 7.4), the brain sections were incubated with primary antibody
(Rabbit Anti-copGFP 1:5000; Evrogen) mixed in sterile PBS, $0.1 \%$ Triton $\mathrm{X}-100$ and $1 \%$ BSA for $2 \mathrm{~h}$ at room temperature. The brain sections were rinsed in sterile PBS and incubated with biotinylated secondary antibody (Donkey Anti-Rabbit 1:1500; Jackson Laboratories) mixed in PBS, $0.3 \%$ Triton $\mathrm{X}-100$ and $1 \%$ BSA for $1 \mathrm{~h}$ at room temperature. Sections were then rinsed with PBS and incubated at room temperature with the avidin-biotinperoxidase complex (Vectastain Elite; Vector Laboratories, Burlingame, CA) mixed in PBS, $0.1 \%$ Triton X-100 for $1 \mathrm{~h}$ 30 min. After several sterile rinses in sterile PBS, brain sections were immersed in a mixture containing sterile PBS, the chromagen $3,3^{\prime}$-diaminobenzidine tetrahydrochloride $(0.05 \%)$, and $\mathrm{H}_{2} \mathrm{O}_{2}(0.003 \%)$. Sections were rinsed in sterile PBS, mounted onto positively charged slides (Surgipath, Richmond, IL) and followed by an in situ hybridization against ENK to quantify the knockdown of ENK in the BLAp (representative images are presented in Figure $4 \mathrm{a}$ ).

Statistical analysis. All data is presented as means and standard errors. Data was analyzed using Aabel, version 3, software (Gigawiz). Animal grouping was performed by using the k-means clustering analysis of the four principal components evaluated in behavioral tests (duration of social interaction, sucrose intake, total distance traveled in EPM, and open-arms time ratio in EPM). Student's $t$-test has been used for control vs CUS and for scrambled $v s$ ENK shRNA analysis. One-way analysis of variance followed by a TukeyKramer post hoc has been used for control $v s$ resilient $v s$ vulnerable analysis. Two-way analysis of variance has been used for the analysis of repeated measures with unbalanced designs. Unbalanced design was used because of the unequal number of subjects per groups. Analysis was performed by an investigator blind to the treatment group of the subjects. Analysis was deemed significant at $p<0.05$.

\section{RESULTS}

\section{Experiment 1}

Resilience and vulnerability to CUS. To investigate whether rats experiencing CUS developed anxiety-like physiological and behavioral responses, all the animals were subjected to several measurements during the experimentation. Animals were randomly assigned to stress $(n=48)$ or unstress (control; $n=25)$ groups. Both groups were similar for weight, corticosterone levels, sucrose intake and preference, and social interaction at the beginning of the CUS. To evaluate the neurobiological process underlying individual differences in reactivity to CUS, stressed animals were divided into two clusters. The unsupervised k-means clustering analysis was applied to the four principal behavioral components in order to reduce bias in the selection of resilient and vulnerable individuals. The behavioral parameters were: (1) duration of social interaction, (2) sucrose intake, (3) total distance traveled in EPM, and (4) open-arms time ratio in EPM. This choice was based on the principal component analysis of all behavioral responses that revealed that the duration of social interaction was the main factor and explained $65.34 \%$ of the variability between animals, open/total ratio in the EPM, $32.83 \%$, total arm distance in the EPM, $1.59 \%$, and sucrose 


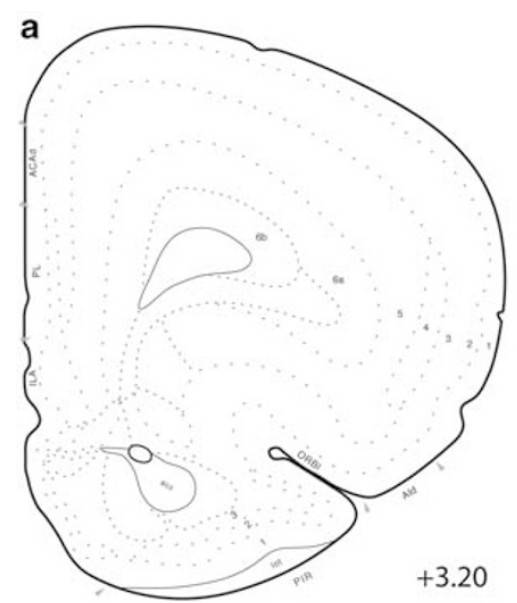

b
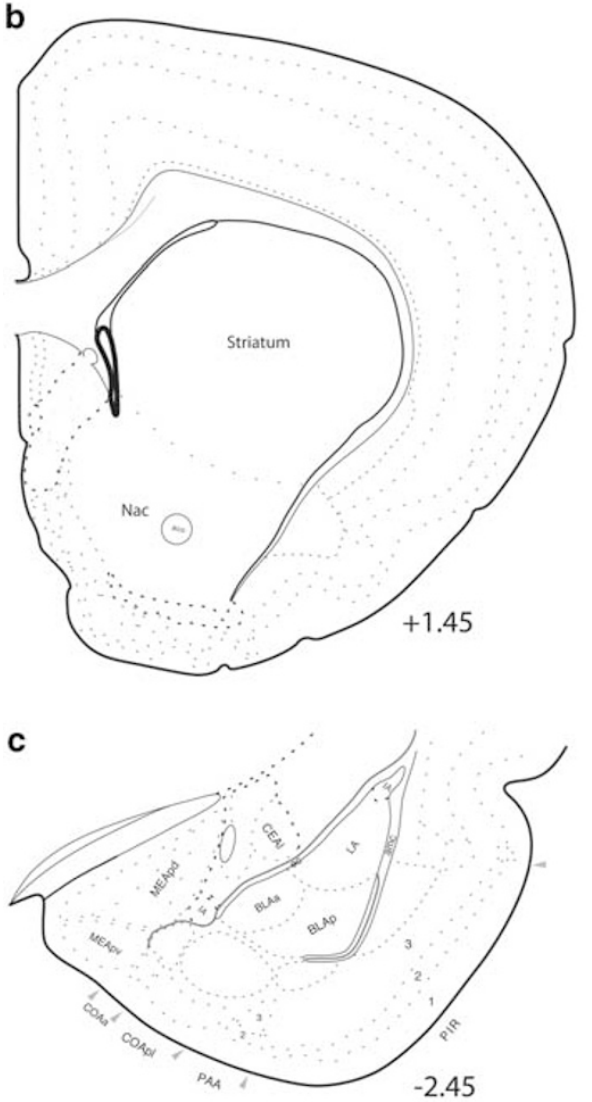

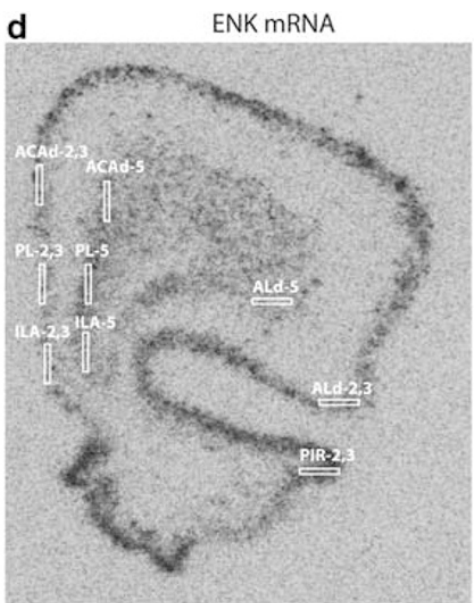

e

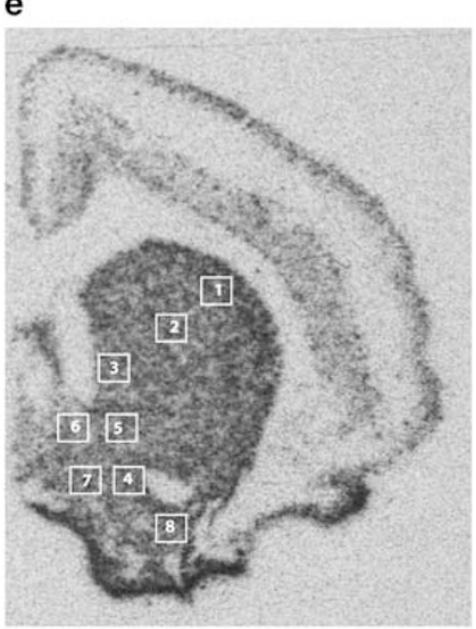

f

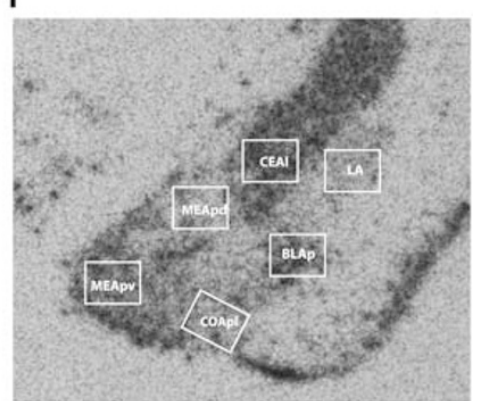

Figure I Representative autoradiographs for enkephalin (ENK) mRNA within the medial prefrontal cortex, nucleus accumbens (NAc), dorsal striatum and amygdaloid complex with corresponding bregma coordonates (a-c) (Swanson, 2003). (d) ACAd, anterior cingulate area, dorsal part; ILA, infralimbic area; PL, prelimbic area; Ald, agranular insular area, dorsal part; PIR, piriform area. (e) I- striatum, dorsolateral part; 2- striatum, central part; 3- striatum, medial part; 4- NAc, ventral core; 5- NAc, dorsal core; 6- NAc, dorsal shell; 7- NAc, medial shell; NAc, lateral shell. (f) CeAl, central amygdalar nucleus, lateral part; LA, lateral amygdalar nucleus; MEApd, medial amygdalar nucleus, posterodorsal part; MEApv, medial amygdalar nucleus, posteroventral part; COApl, cortical amygdalar nucleus, posterior part, lateral zone; BLAp, basolateral amygdalar nucleus, posterior part.

intake, $0.25 \%$. Clustering analysis yielded clearly discrete clusters, and the centroids (mean clusters) were significantly different from each other (Figure 2). The first cluster $(n=29)$ was characterized by a lower duration of social interaction $\left(F_{(2,71)}=46.7, p<0.001\right.$; Figure 3a), lower openarms time ratio in $\operatorname{EPM}\left(F_{(2,72)}=8.5, p<0.001\right.$; Figure $\left.3 \mathrm{e}\right)$ and lower total arm distance in $\operatorname{EPM~}\left(F_{(2,72)}=5.9, p=0.004\right.$; Figure $3 \mathrm{~d})$ when compared with the second cluster $(n=19)$.
The first cluster was also different from the second cluster by having higher sucrose intake 7 days after the last stressor $\left(F_{(2,72)}=5.6, p=0.006\right.$; Figure $\left.3 \mathrm{~g}\right)$, and lower restraint-induced corticosterone level at days $11\left(F_{(2,67)}=291, p<0.001\right.$; Figure $3 c)$ and $21\left(F_{(2,68)}=183, p<0.001\right.$; Figure $\left.3 c\right)$. When compared with control individuals, the first cluster showed lower duration of social interaction $\left(F_{(2,71)}=46.7, p<0.001\right.$; Figure $3 \mathrm{a})$, weight gain, $\left(F_{(2,72)}=49.2 p<0.001\right.$; Figure $\left.3 \mathrm{~b}\right)$, 


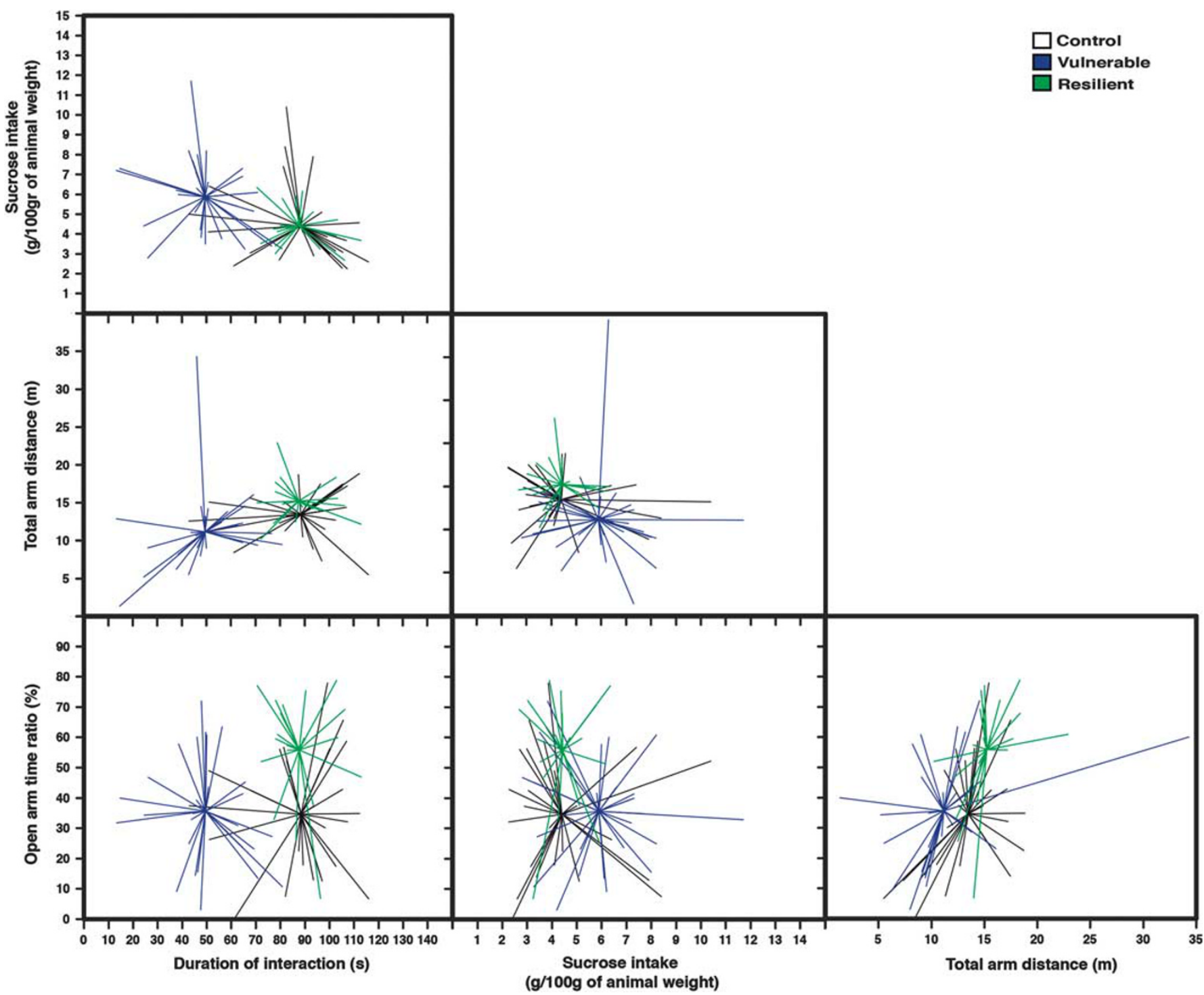

Figure 2 Matrix scatter diagram displaying three groups that have been differentiated by k-means analysis. This was done by a partition of four behavioral parameters into three clusters (duration of social interaction, open-arms time ratio in the elevated plus maze (EPM), total arm distance traveled in the EPM and sucrose intake). A line connects each point to its cluster centroid. The black cluster represents control $(n=25)$, the blue cluster represents vulnerable $(n=29)$ and the green cluster represents resilient $(n=19)$ individuals.

higher basal corticosterone at day $21\left(F_{(2,67)}=4.4, p=0.016\right.$; Figure $3 \mathrm{c})$, and sucrose intake $\left(F_{(2,72)}=5.6, p=0.006\right.$; Figure 3g). The second cluster showed similar response when compared with control rats for time of social interaction, number of social interactions, sucrose intake, sucrose preference, basal corticosterone levels, and total arm distance in EPM. The second cluster showed only a difference from control animals by having lower weight gain $\left(F_{(2,72)}=49.2 p<0.001\right.$; Figure $\left.3 \mathrm{~b}\right)$ and higher open-arms time ratio in $\operatorname{EPM},\left(F_{(2,72)}=8.5, p<0.001\right.$; Figure $\left.3 \mathrm{e}\right)$. No difference was observed between groups in the sucrose preference test (Figure $3 \mathrm{~h}$ ). Overall, the first cluster displayed more anxiety-like behavior and passive coping strategy compared with the second cluster and control animals, and was identified as being vulnerable. The second cluster reflects more similarities with control animals, showing a better adaptation than the first cluster to CUS, and was identified as being resilient.
ENK $m R N A$ expression is associated with resilience and vulnerability to CUS. ENK mRNA expression have been quantified in sub-divisions of prefrontal cortex, dorsal striatum, NAc, and amygdaloid complex (Figure 1). Among all the quantified areas, ENK mRNA expression, 7 days after the exposure to the last stressor, was decreased by $36 \%$ in the BLAp in vulnerable compared with control and resilient individuals $\left(F_{(2,68)}=26.79, p<0.001\right.$; Figure $\left.3 f\right)$. In contrast, there was no difference in ENK expression in BLAp between control and resilient. There was no difference between control, resilient, and vulnerable in all the other quantified areas (Supplementary Table S2).

\section{Experiment 2}

shRNA vector validation of ENK knockdown efficiency in the BLAp. To evaluate the modulation of anxiety behaviors by $\mathrm{ENK}$ in the BLAp, 15 rats received bilateral 
a

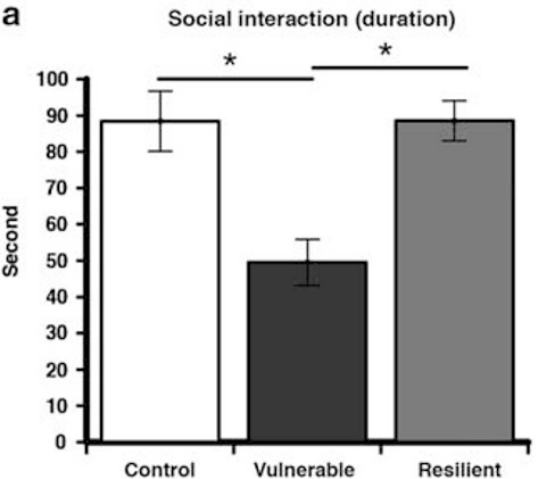

b
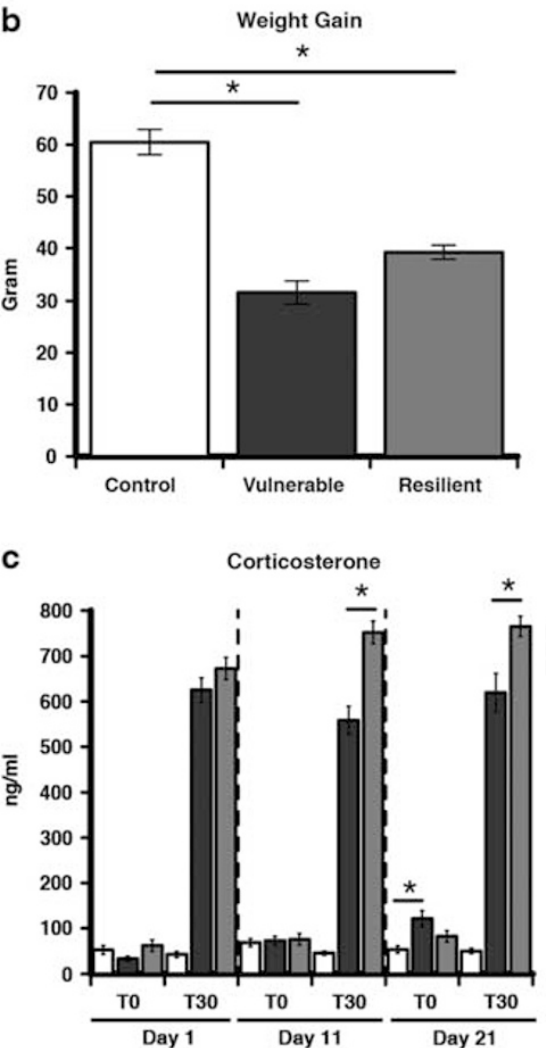

d



e

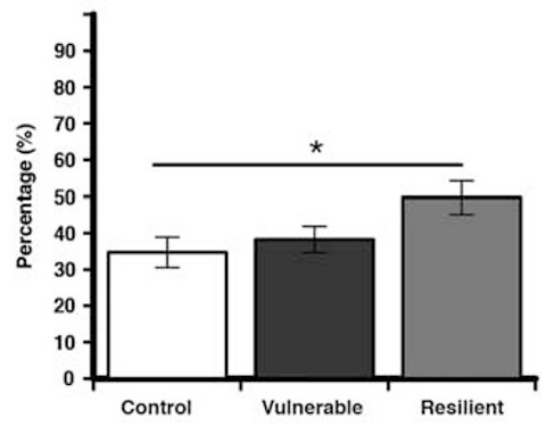

g

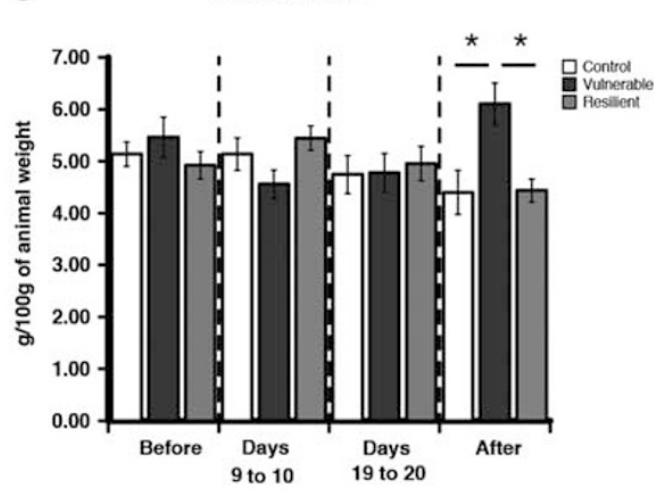

h



Figure 3 Resilience and vulnerability to 21 days of chronic unpredictable stress (CUS) are marked by specific long-term behavioral, physiological and neuroadaptation in the enkephalinergic (ENKergic) brain circuit. (a) Vulnerable displayed lower duration of social interaction compared with control and resilient individuals. (b) Vulnerable showed lower weight gain compared with resilient individuals. (c) Vulnerable individuals showed higher basal corticosterone compared with control individuals. (d) Vulnerable displayed decreased locomotion as measured by total arm distance in the elevated plus maze (EPM) compared with resilient individuals. (e) Resilient displayed higher exploration as measured by the open-arm time ratio in the EPM compared with control. (f) Enkephalin (ENK) mRNA is reduced only in posterior part of the amygdalar nucleus (BLAp) in vulnerable compared with control and resilient individuals I week after the last stress session. In contrast there is no difference between control and resilient individuals. (g) Vulnerable displayed enhanced sucrose consumption compared with control and resilient individuals. (h) No difference in the sucrose preference test between groups. Social interaction was assessed 3 days after the end the CUS. EPM was evaluated $24 \mathrm{~h}$ after the social interaction. Sucrose intake and preference tests were performed before (days -3 to -1 ), during (days 9-10 and 19-20), and after (days 4-6) the CUS. Bars represent mean \pm SEM; * $<<0.05$.

injections of the ENK shRNA lentiviral vector, whereas 8 rats received bilateral injections of the scrambled shRNA lentiviral vector. Examination of immunolabeled copGFP distribution under darkfield microscopy by an experimenter unaware of behavioral results revealed that 21 animals had injection sites centered bilaterally in the BLAp, with the lateral part of the central amygdala (CeAl), the lateral amygdala, and intercalated cell mass mostly untouched by injections (Figure 4a). Two animals were removed from the analysis because their injection sites were only successfully bilaterally targeted. These animals with viral injections bilaterally centered in the BLAp with shRNA targeting ENK $(n=13)$ and scrambled shRNA $(n=8)$ were included for behavioral analysis. The injection sites were centered within high ENK expressing neurons of the BLAp. To validate the efficiency of our lentiviral system, we compared ENK mRNA expression in scrambled and ENK shRNA groups. We observed a 59\% reduction of ENK mRNA level in the 
a
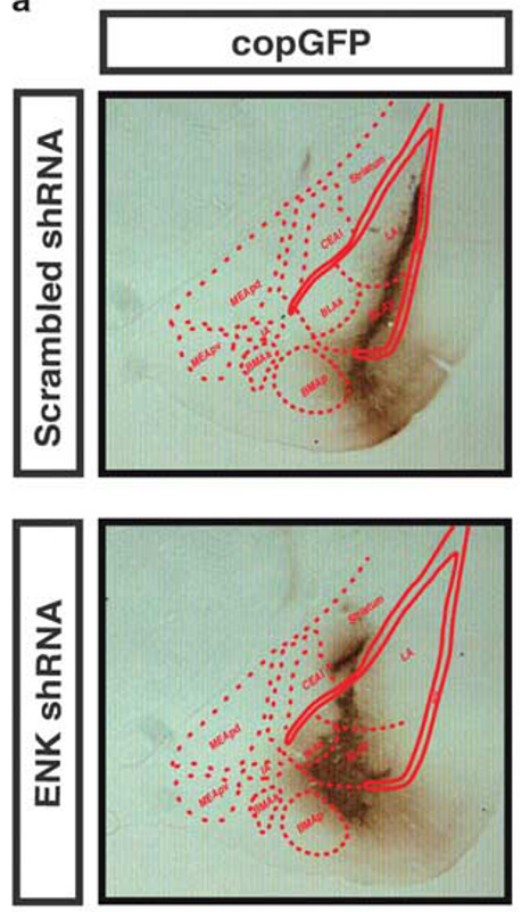

C
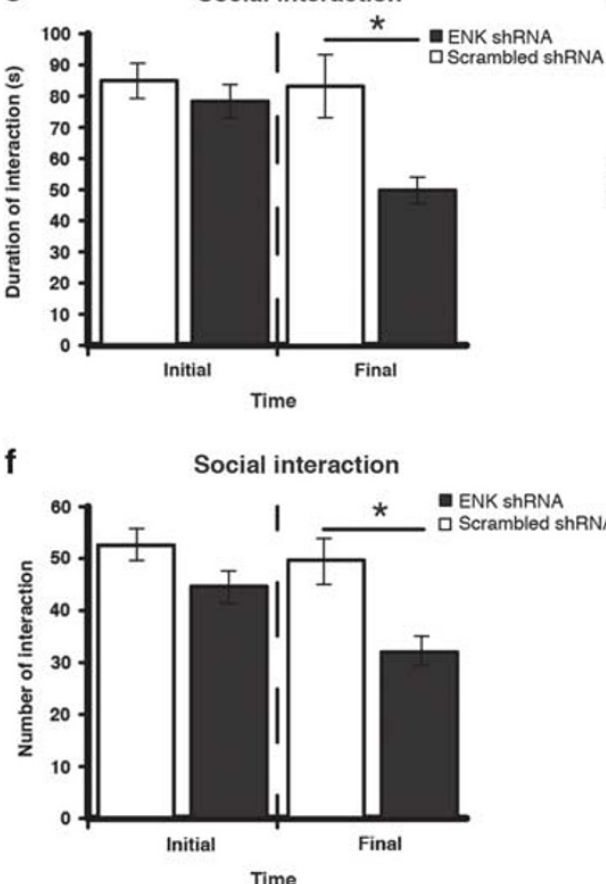
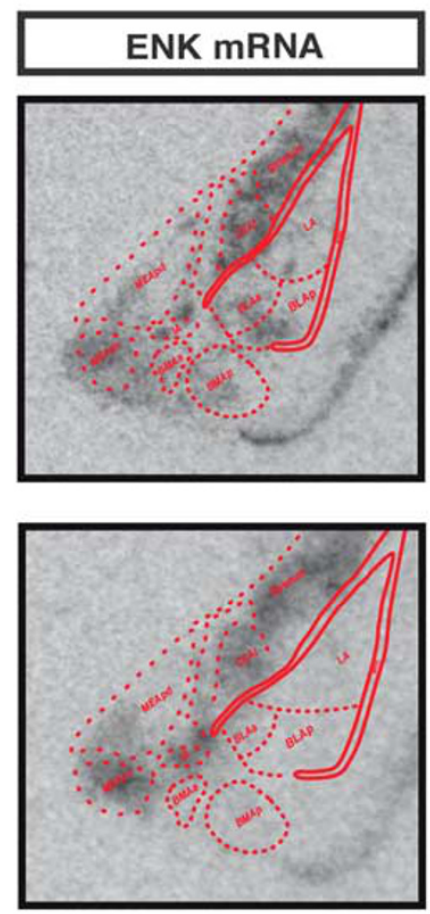

d



g

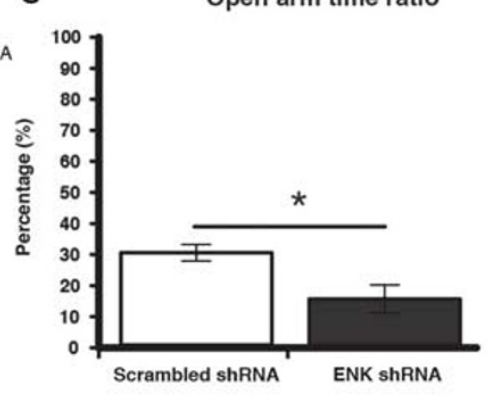

b


h Sucrose preference

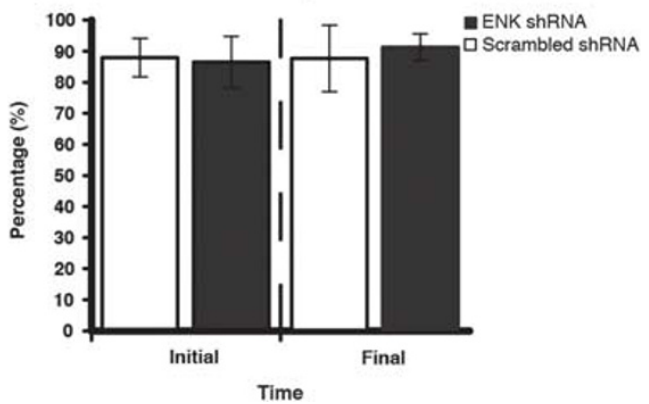

Figure 4 Enkephalin (ENK) knockdown increased anxiety-like behaviors. (a) Representative immunoreactive for copepod green fluorescent protein (copGFP) and autoradiographs for ENK mRNA within the amygdaloid complex. (b) We observed a 59\% reduction of ENK mRNA level in ENK shRNA animals when compared with scrambled shRNA animals. ENK knockdown decrease the duration of social interaction (c); the number of social interaction (f); and the open-arm time ratio in the elevated plus maze (EPM) (g). ENK knockdown did not affect the total arm distance in the EPM (d); the sucrose intake (e); and the sucrose preference $(h)$. Social interaction, sucrose intake, and sucrose preference tests were evaluated in the same animals before the administration of the viral vector (initial) and after the 14 days of the recovery period (final). Bars represent mean \pm SEM ( $n=13$ for ENK and scrambled $n=8)$; * $p<0.05$.

BLAp of ENK shRNA animals when compared with scrambled shRNA animals $\left(t_{(19)}=5.85, p<0.001\right.$; Figure $\left.4 \mathrm{~b}\right)$ without affecting ENK mRNA in the CeAl $\left(t_{(19)}=0.96\right.$, $p=0.349)$ and lateral amygdala $\left(t_{(19)}=0.27, \mathrm{p}>0.5\right.$; data not shown).
ENK knockdown in the posterior BLAp decreases social interaction and open-arm exploration. Animals injected with lentiviral vectors were submitted sequentially, $24 \mathrm{~h}$ apart, to the social interaction, EPM and sucrose intake and preference tests. The knockdown of ENK in the BLAp 
significantly decreased the duration $\left(t_{(19)}=3.54, p=0.002\right.$; Figure $4 c)$ and the number $\left(t_{(19)}=3.55, p=0.002\right.$; Figure $4 \mathrm{f}$ ) of social interaction compared with the scrambled shRNA group. In the EPM, knockdown of ENK in the BLAp significantly decreased the open/total ratio compared with scrambled shRNA $\left(t_{(19)}=2.39, p<0.027\right.$; Figure $\left.4 \mathrm{~g}\right)$, without affecting the total arm distance (Figure 4d), which suggests a higher exploratory activity with no general locomotor effect. However, analysis of the sucrose intake or preference revealed no significant effect (Figure $4 \mathrm{e}$ and $\mathrm{h}$ ). Overall, the knockdown of ENK in the BLAp had anxiogenic properties and reproduces some aspects found in vulnerable individuals to CUS.

\section{DISCUSSION}

The present study shows the extent to which adaptive changes in the ENK system is important mediator underlying resilience to CUS. The results emerging from this work demonstrate that there are important and enduring differences in the magnitude of many aspects of the stress responses among a homogenous population of rats exposed to the same stressful condition. Based on the k-mean analysis of the wide distribution of the behavioral responses, stressed individuals were split into two subpopulations. Individuals with similar behavioral responses to control were labeled resilient, and those with displayed anxiety-like behavior were labeled vulnerable. Interestingly, vulnerable and resilient exhibited distinct basal corticosterone levels as well as restraint-induced corticosterone release. Both subpopulations displayed similar basal corticosterone levels and similar restraint-induced corticosterone release at day 1 of the CUS. However, vulnerable displayed lower corticosterone release during the subsequent restraint sessions at days 11 and 21 compared with resilient, and only vulnerable exhibit enhance basal corticosterone at the end of CUS (day 21) compared with control individuals. The release of corticosterone, as well as its inhibition, is mediated by complex mechanisms that control the activation/inhibition of the hypothalamo-pituitary-adrenocortical axis (Herman, 2003). Facilitation of the hypothalamo-pituitary-adrenocortical activation in response to a novel stress appears to be a key feature of resilience (Wood et $a l, 2010$ ) as well as the capacity to maintain low basal level of corticosterone when the stress ceases (Feder et al, 2009). In contrast, having persistent high corticosterone levels is an important risk factor to stressrelated psychiatric disorders like depression (Nemeroff and Vale, 2005).

\section{Decreased BLAp ENK: a Molecular Signature of Vulnerability}

The present study demonstrates persistent CUS-induced changes in the ENKergic neuronal systems, which seem to dictate to occurring variations in physiological and behavioral outcomes. The BLAp ENK levels were only decreased in vulnerable individuals. Recently, we published findings demonstrating that ENK levels were also specifically decreased in the BLAp of vulnerable individuals to chronic social defeat stress (Bérubé et al, 2013). This allowed us to demonstrate that the reduction of ENK mRNA expression in the BLAp of vulnerable individuals is a repeatable effect found in two different chronic stress models. These results are also consistent with the wellknown anxiolytic properties of the ENKergic neurotransmission. The ENK through the activation of $\mu-O R$ and/or $\delta$-OR may attenuate stress-induced neuroendocrine, autonomic, and anxiety-like behavioral responses (König et al, 1996; Kang et al, 2000; Filliol et al, 2000; Ragnauth et al, 2001; Drolet et al, 2001; Hebb et al, 2004; Hebb et al, 2005a; Hebb et al, 2005b; Jutkiewicz, 2007; Noble et al, 2007). In addition, pharmacological studies reported that systemic administration of $\mu$-OR or $\delta$-OR agonists have anxiolytic and antidepressant properties in rodents (Broom et al, 2002; Saitoh et al, 2004; Perrine et al, 2006; Vergura et al, 2008; Rezayof et al, 2009).

The BLA is a critical brain region for the generation of emotional behavior and produces appropriate emotional responses by activating a large number of brain regions. Consistent with our findings, some studies revealed that the BLA is a key brain region implicated in the resilience following acute or chronic restraint stress (Grissom and Bhatnagar, 2011; Sajdyk et al, 2008). These studies revealed that resilience is mediated by adaptive changes in the BLA, which involve numerous neurotransmitters. Previous pharmacological and lesion studies indicated that BLA activation increases, whereas its inhibition decreases anxiety-like behavior and stress-induced physiological responses (Sanders and Shekhar, 1995; Sajdyk and Shekhar, 1997; Thielen and Shekhar, 2002; Truitt et al, 2009). It is noteworthy that the efferents of the BLA arise from pyramidal cells (McDonald, 1992) and the majority $(98 \%)$ of ENKergic cells in the BLA are glutamatergic pyramidal-like cells in BLAp (Poulin et al, 2008). The BLA activates a large number of brain regions by sending direct efferents to the CeA, the intercalated cells of the amygdala, the bed nucleus of the stria terminalis, the medial prefrontal cortex, the NAc, and the dorsal striatum (Dong et al, 2001; Gabbott et al, 2006; McDonald, 1991; Royer et al, 1999; St Onge et al, 2012; Wright et al, 1996). It is currently unclear which pathway is responsible for the effects reported here because the BLA ENKergic efferents are poorly described. Only the ENKergic efferents to the CeAm have been reported (Poulin et al, 2006). Also, pharmacological manipulation of OR in the CeA was show to modulate anxiety-like behaviors in the social interaction and EPM tests (File and Rodgers, 1979; Rodgers and File, 1979; Zarrindast et al., 2008; Wilson and Junor, 2008). However, these studies revealed conflicting results in rodent, both anxiogenic and anxiolytic effects have been reported. In contrast to pharmacological studies, the viral approach described here assess the phenotype associated with a depletion of ENK in depicted area of the BLAp, allows a cellular resolution of the neurons transduced and depleted in ENK, without affecting the potential role of ENK release from other brain structures. Electrophysiological studies revealed that BLA stimulation induced an excitatory postsynaptic current in CeA neurons ( $\mathrm{Zhu}$ and Pan, 2004), and that $\mu$-OR activation inhibits glutamate excitatory postsynaptic current primarily by reducing presynaptic glutamate release in the CeA (Zhu and Pan, 2005). Therefore, the ability to maintain ENK levels in the BLA following chronic stress exposure 
may facilitate resilience by promoting the capacity to modulate appropriately the release of glutamate in the BLA output regions.

Although recent studies have demonstrated without a doubt the importance of the BLA in the control of anxiety behavior in the open-arm maze (Felix-Ortiz et al, 2013; Tye et al, 2011), the BLA is not the unique neural substrate of anxiety behavior. Indeed, numerous brain regions have previously been implicated in anxiety-like behavior including the CeAl, septum, and hippocampus (for a review McEwen et al 2012) Our data indicate a possible interaction between the CUS and ENK mRNA in the effect on the EPM. Indeed, some studies have demonstrated that stress can increase exploration of the EPM as measure by locomotor activity (for instance see Strekalova and Steinbusch 2010). Our data seem to indicate that this effect of stress on exploratory activity is independent of ENK in BLA, and could oppose the effect observed in the ENK mRNA knockdown experiment.

In addition to the ENKergic dichotomy found in resilient and vulnerable individuals, the functional role of BLAp ENKergic neurons was assessed in the present study, and demonstrates that ENK knockdown is sufficient to increase anxiety and reproduce in unstressed rats the major behavioral disturbances found in vulnerable individuals. The ENK knockdown demonstrates that ENK depletion in the BLAp leads to behavioral disturbances such as an increase of anxiety in the social interaction and EPM tests. The failure of compensation for ENK expression found in the BLAp in vulnerable individuals following a CUS, combined with the anxiogenic properties of the ENK knockdown in the BLAp, support the hypothesis that resilience to chronic stress may be mediated in part by adaptive mechanisms that permit ENK recovery in the BLA at the level of control individuals. Further analysis of the BLAp ENKergic circuits and signaling mechanisms will be important to identify the fundamental mechanisms governing resilience to chronic stress.

Unfortunately, we do not have the initial social interaction data for all the rats. However, when initial social interaction data was available before CUS, rats were matched between group accordingly. However, we cannot exclude the possibility that behavioral variability in these groups was present before stress and not a product of the manipulation. Moreover, we did some control experiments to test whether the sucrose habituation for 14 days was changing the ENK expression before the beginning of CUS protocol. One group $(n=12)$ had access to one bottle of water and one bottle of sucrose for 14 days, whereas the other group $(n=12)$ had access to two bottles of water. We did not see any difference in the ENK expression in the BLAp or in the time of social interaction.

The CUS model should prove useful for further investigation of the neurobiological mechanisms underlying cognitive and behavioral consequences of chronic stress exposure that may be important in the development of anxiety disorders. These results have broad implications for understanding the functional role of ENKergic neurotransmission following repeated unpredictable psychogenic stress, and suggest that ENK release facilitates the adaptation of behavioral responses to chronic stress.

\section{FUNDING AND DISCLOSURE}

This research was funded by the Canadian Institutes of Health Research (CIHR), grant number MOP-62921. PB was supported by a CIHR (Banting \& Best) studentship and J-FP by an NSERC studentship.

\section{REFERENCES}

Bergstrom A, Jayatissa M, Mork A, Wiborg O (2008). Stress sensitivity and resilience in the chronic mild stress rat model of depression; an in situ hybridization study. Brain Res 1196: 41-52.

Bérubé P, Laforest S, Bhatnagar S, Drolet G (2013). Enkephalin and dynorphin mRNA expression are associated with resilience or vulnerability to chronic social defeat stress. Physiol Behav 122: 237-245.

Bondi CO, Rodriguez G, Gould GG, Frazer A, Morilak DA (2007). Chronic unpredictable stress induces a cognitive deficit and anxiety-like behavior in rats that is prevented by chronic antidepressant drug treatment. Neuropsychopharmacology 33: 320-331.

Broom DC, Jutkiewicz EM, Folk EF, Traynor JR, Rice KC, Woods JH (2002). Nonpeptidic $\delta$-opioid receptor agonists reduce immobility in the forced swim assay in rats. Neuropsychopharmacology 26: 744-755.

Christiansen S, Bouzinova EV, Palme R, Wiborg O (2012). Circadian activity of the hypothalamic-pituitary-adrenal axis is differentially affected in the rat chronic mild stress model of depression. Stress 15: 647-657.

Covington HE, Lobo MK, Maze I, Vialou V, Hyman JM, Zaman S et al (2010). Antidepressant effect of optogenetic stimulation of the medial prefrontal cortex. J Neurosci 30: 16082-16090.

Dong HW, Petrovich GD, Swanson LW (2001). Topography of projections from amygdala to bed nuclei of the stria terminalis. Brain Res Brain Res Rev 38: 192-246.

Drolet G, Dumont EC, Gosselin I, Kinkead R, Laforest S, Trottier JF (2001). Role of endogenous opioid system in the regulation of the stress response. Prog Neuropsychopharmacol Biol Psychiatry 25: 729-741.

Dumont EC, Kinkead R, Trottier JF, Gosselin I, Drolet G (2000). Effect of chronic psychogenic stress exposure on enkephalin neuronal activity and expression in the rat hypothalamic paraventricular nucleus. J Neurochem 75: 2200-2211.

Feder A, Nestler EJ, Charney DS (2009). Psychobiology and molecular genetics of resilience. Nat Rev Neurosci 10: 446-457.

Felix-Ortiz AC, Beyeler A, Seo C, Leppla CA, Wildes CP, Tye KM (2013). BLA to vHPC inputs modulate anxiety-related behaviors. Neuron 79: 654-664.

File SE, Rodgers RJ (1979). Exploratory behaviour and aversive thresholds in rats following microinjection of morphine into central and medial nuclei of the amygdala [proceedings]. British Journal of Pharmacology 66: 145-146.

Filliol D, Ghozland S, Chluba J, Martin M, Matthes HW, Simonin F et al (2000). Mice deficient for delta- and mu-opioid receptors exhibit opposing alterations of emotional responses. Nat Genet 25: 195-200.

Gabbott PLA, Warner TA, Busby SJ (2006). Amygdala input monosynaptically innervates parvalbumin immunoreactive local circuit neurons in rat medial prefrontal cortex. NSC 139: 1039-1048.

Grissom NM, Bhatnagar S (2011). The basolateral amygdala regulates adaptation to stress via $\beta$-adrenergic receptormediated reductions in phosphorylated extracellular signalregulated kinase. Neuroscience 178: 108-122.

Hebb A, Drolet G, Mendella P, Roach S, Gauthier M, Zacharko R (2005a). Intracerebroventricular d-Pen2, d-Pen5-enkephalin administration soon after stressor imposition influences 
behavioral responsivity to a subsequent stressor encounter in CD-1 mice. Pharmacol Biochem Behav 82: 453-469.

Hebb A, Poulin J, Roach S, Zacharko R, Drolet G (2005b). Cholecystokinin and endogenous opioid peptides: Interactive influence on pain, cognition, and emotion. Prog NeuroPsychopharmacol Biol Psychiatry 29: 1225-1238.

Hebb A, Zacharko RM, Gauthier M, Trudel F, Laforest S, Drolet G (2004). Brief exposure to predator odor and resultant anxiety enhances mesocorticolimbic activity and enkephalin expression in CD-1 mice. Eur J Neurosci 20: 2415-2429.

Herman J (2003). Central mechanisms of stress integration: hierarchical circuitry controlling hypothalamo-pituitary-adrenocortical responsiveness. Front Neuroendocrinol 24: 151-180.

Jutkiewicz EM (2007). RB101-mediated protection of endogenous opioids: potential therapeutic utility? CNS Drug Rev 13: 192-205.

Kang W, Wilson SP, Wilson MA (2000). Overexpression of proenkephalin in the amygdala potentiates the anxiolytic effects of benzodiazepines. Neuropsychopharmacology 22: 77-88.

König M, Zimmer AM, Steiner H, Holmes PV, Crawley JN, Brownstein MJ et al (1996). Pain responses, anxiety and aggression in mice deficient in pre-proenkephalin. Nature 383: 535-538.

Krishnan V, Han M-H, Graham DL, Berton O, Renthal W, Russo SJ et al (2007). Molecular adaptations underlying susceptibility and resistance to social defeat in brain reward regions. Cell 131: 391-404.

McDonald AJ (1991). Topographical organization of amygdaloid projections to the caudatoputamen, nucleus accumbens, and related striatal-like areas of the rat brain. Neuroscience 44: 15-33.

McDonald AJ (1992). Projection neurons of the basolateral amygdala: a correlative Golgi and retrograde tract tracing study. Brain Res Bull 28: 179-185.

McEwen BS, Eiland L, Hunter RG, Miller MM (2012). Stress and anxiety: structural plasticity and epigenetic regulation as a consequence of stress. Neuropharmacology 62: 3-12.

Nemeroff CB, Vale WW (2005). The neurobiology of depression: inroads to treatment and new drug discovery. J Clin Psychiatry 66(Suppl 7): 5-13.

Noble F, Benturquia N, Bilkei-Gorzo A, Zimmer A, Roques BP (2007). Use of preproenkephalin knockout mice and selective inhibitors of enkephalinases to investigate the role of enkephalins in various behaviours. Psychopharmacology 196: 327-335.

Perrine SA, Hoshaw BA, Unterwald EM (2006). Delta opioid receptor ligands modulate anxiety-like behaviors in the rat. $\mathrm{Br} J$ Pharmacol 147: 864-872.

Poulin J-F, Bérubé P, Laforest S, Drolet G (2013). Enkephalin knockdown in the central amygdala nucleus reduces unconditioned fear and anxiety. Eur J Neurosci 37: 1357-1367.

Poulin J-F, Castonguay-Lebel Z, Laforest S, Drolet G (2008). Enkephalin co-expression with classic neurotransmitters in the amygdaloid complex of the rat. J Comp Neurol 506: 943-959.

Poulin J-F, Chevalier B, Laforest S, Drolet G (2006). Enkephalinergic afferents of the centromedial amygdala in the rat. J Comp Neurol 496: 859-876.

Ragnauth A, Schuller A, Morgan M, Chan J, Ogawa S, Pintar J et al (2001). Female preproenkephalin-knockout mice display altered emotional responses. Proc Natl Acad Sci USA 98: 1958-1963.

Rezayof A, Hosseini SS, Zarrindast MR (2009). Effects of morphine on rat behaviour in the elevated plus maze: The role of central amygdala dopamine receptors. Behav Brain Res 202: 171-178.

Rodgers RJ, File SE (1979). Exploratory behaviour and aversive thresholds following intra-amygdaloid application of opiates in rats. Pharmacol Biochem Behav 11: 505-511.

Royer S, Martina M, Pare D (1999). An inhibitory interface gates impulse traffic between the input and output stations of the amygdala. J Neurosci 19: 10575-10583.
Saitoh A, Kimura Y, Suzuki T, Kawai K, Nagase H, Kamei J (2004). Potential anxiolytic and antidepressant-like activities of SNC80, a selective $\delta$-opioid agonist, in behavioral models in rodents. J Pharmacol Sci 95: 374-380.

Sajdyk TJ, Johnson PL, Leitermann RJ, Fitz SD, Dietrich A, Morin $\mathrm{M}$ et al (2008). Neuropeptide $\mathrm{Y}$ in the Amygdala induces longterm resilience to stress-induced reductions in social responses but not hypothalamic-adrenal-pituitary axis activity or hyperthermia. J Neurosci 28: 893-903.

Sajdyk TJ, Shekhar A (1997). Excitatory amino acid receptors in the basolateral amygdala regulate anxiety responses in the social interaction test. Brain Res 764: 262-264.

Sanders SK, Shekhar A (1995). Regulation of anxiety by GABAA receptors in the rat amygdala. Pharmacol Biochem Behav 52: 701-706.

St Onge JR, Stopper CM, Zahm DS, Floresco SB (2012). Separate prefrontal-subcortical circuits mediate different components of risk-based decision making. J Neurosci 32: 2886-2899.

Strekalova T, Steinbusch HW (2010). Mesuring behavior in mice with chronic stress paradigm. Prog Neuropsychopharmacol Biol Psychiatry 34: 348-361.

Swanson LW (2003). Brain Maps III: Structure of the Rat Brain. Elsevier Science \& Technology Books.

Taliaz D, Loya A, Gersner R, Haramati S, Chen A, Zangen A (2011). Resilience to chronic stress is mediated by hippocampal brainderived neurotrophic factor. J Neurosci 31: 4475-4483.

Thielen SK, Shekhar A (2002). Amygdala priming results in conditioned place avoidance. Pharmacol Biochem Behav 71: 401-406.

Truitt WA, Johnson PL, Dietrich AD, Fitz SD, Shekhar A (2009). Anxiety-like behavior is modulated by a discrete subpopulation of interneurons in the basolateral amygdala. Neuroscience 160: 284-294.

Tye KM, Prakash R, Kim SY, Fenno LE, Grosenick L, Zarabi H et al (2011). Amygdala circuitry mediating reversible and bidirectional control of anxiety. Nature 471: 358-362.

Vergura R, Balboni G, Spagnolo B, Gavioli E, Lambert DG, McDonald J et al (2008). Anxiolytic- and antidepressantlike activities of H-Dmt-Tic-NH-CH(CH2-COOH)-Bid (UFP512), a novel selective delta opioid receptor agonist. Peptide 29: 93-103.

Wang C, Wu H-M, Jing X-R, Meng Q, Liu B, Zhang H et al (2012). Oxidative parameters in the rat brain of chronic mild stress model for depression: relation to anhedonia-like responses. $J$ Membr Biol 245: 675-681.

Wilson MA, Junor L (2008). The role of amygdalar mu-opioid receptors in anxiety-related responses in two rat models. Neuropsychopharmacology 33: 2957-2968.

Wood SK, Walker HE, Valentino RJ, Bhatnagar S (2010). Individual differences in reactivity to social stress predict susceptibility and resilience to a depressive phenotype: role of corticotropin-releasing factor. Endocrinology 151: 1795-1805.

Wright CI, Beijer AV, Groenewegen HJ (1996). Basal amygdaloid complex afferents to the rat nucleus accumbens are compartmentally organized. J Neurosci 16: 1877-1893.

Zarrindast M-R, Babapoor-Farrokhran S, Babapoor-Farrokhran S, Rezayof A (2008). Involvement of opioidergic system of the ventral hippocampus, the nucleus accumbens or the central amygdala in anxiety-related behavior. Life Sci 82: 1175-1181.

Zhu W, Pan Z (2004). Synaptic properties and postsynaptic opioid effects in rat central amygdala neurons. Neuroscience 127: 871-879.

Zhu W, Pan Z (2005). $\mu$-opioid-mediated inhibition of glutamate synaptic transmission in rat central amygdala neurons. Neuroscience 133: 97-103.

Supplementary Information accompanies the paper on the Neuropsychopharmacology website (http://www.nature.com/npp) 